\title{
Learning Structural Analysis in "A Building that Teaches"
}

\author{
Katherine Acton \\ University of Minnesota Duluth
}

\begin{abstract}
The Swenson Civil Engineering Building, opened in 2010, was constructed with the goal of providing a space in which, and from which, Civil Engineering students can learn. Multiple exposed structural systems allow students the opportunity to visualize the way in which the building is designed to carry load. The building is LEED certified. A large high-bay lab is open to view, so that class work and research is visible to students as they pass through the halls of the building.
\end{abstract}

The high bay lab features two 15-ton gantry cranes. The gantry cranes have been analyzed in the final project for Structural Analysis (CE 3115). The final project is the culmination of a semester focused on the calculation of loads and deflections in statically determinate and statically indeterminate structures, with an emphasis on beams and frames. The gantry crane project provides an opportunity for students to apply several concepts learned in the text and in lecture. They are required to idealize the three dimensional structure as a two-dimensional structure with support reactions and appropriate connections between the structural members. The students are then asked to solve for the support reaction forces and moments using methods of structural analysis. Students learn to use structural analysis software to check the accuracy of their hand calculations, and to provide further results. Overall, the project is an in-depth analysis of this structural system.

The project is enhanced by the fact that the gantry crane is on site, in the Civil Engineering building, and can be seen in active use as part of other projects. The fact that the crane is a tangible structure, central to the workspace of the department, engages the interest of the students. The crane project represents one aspect of using the newly constructed Civil Engineering building as a "teachable space."

\section{Introduction}

Structural Analysis (CE 3115) is a core course required of all Civil Engineering majors with a focus area Structural Engineering. It is a three credit, junior level course, meeting for 50 minutes three times per week. The course is organized with an average of one graded problem set per week, two midterms, a final project and a final exam.

The Structural Analysis course, and specifically the final project for the course, is designed to develop in students the ability to apply knowledge of mathematics, science and engineering, the ability to communicate effectively, and the ability to use the techniques, skills and modern engineering tools necessary for engineering practice.

Topics covered in the Structural Analysis course that are incorporated in the final project assignment include shear and moment diagrams, influence lines, and methods for calculating support reactions for statically indeterminate structures. Additionally, the 
final project includes an introduction to the modeling software RISA 2D ${ }^{1}$. Modeling software is a modern tool necessary for engineering practice, and one of the goals of the Structural Analysis course is to provide students with an introduction to software that will serve them well in practice, and also in preparation for additional courses such as Finite Element Methods. The students worked in groups of three to create the final project report. This allowed for group discussion of the solutions to each phase of the project, and provided students with an opportunity to develop the skills necessary to work as part of a team.

\section{Gantry Crane Structure}

The Swenson Civil Engineering Building opened for use in the fall of 2010, and is a LEED certified building designed with an emphasis on sustainable use. A notable element of the high bay lab area, shown in Figure 1, is the 15 ton gantry crane. This crane is located on a structural strong floor, and is used in research projects, materials testing and teaching.

A schematic drawing, taken from the preliminary construction drawings for the building project, is shown in Figure 2. Although there are many exposed structural elements in the building to choose from, the gantry crane is a "stand-alone" structure, not connected to other structural elements except for the floor supports. The floor support reactions are calculated as part of the project. The statically indeterminate frame is a commonly analyzed type of structure in the course, which also makes the crane an appealing subject for further examination by the students.

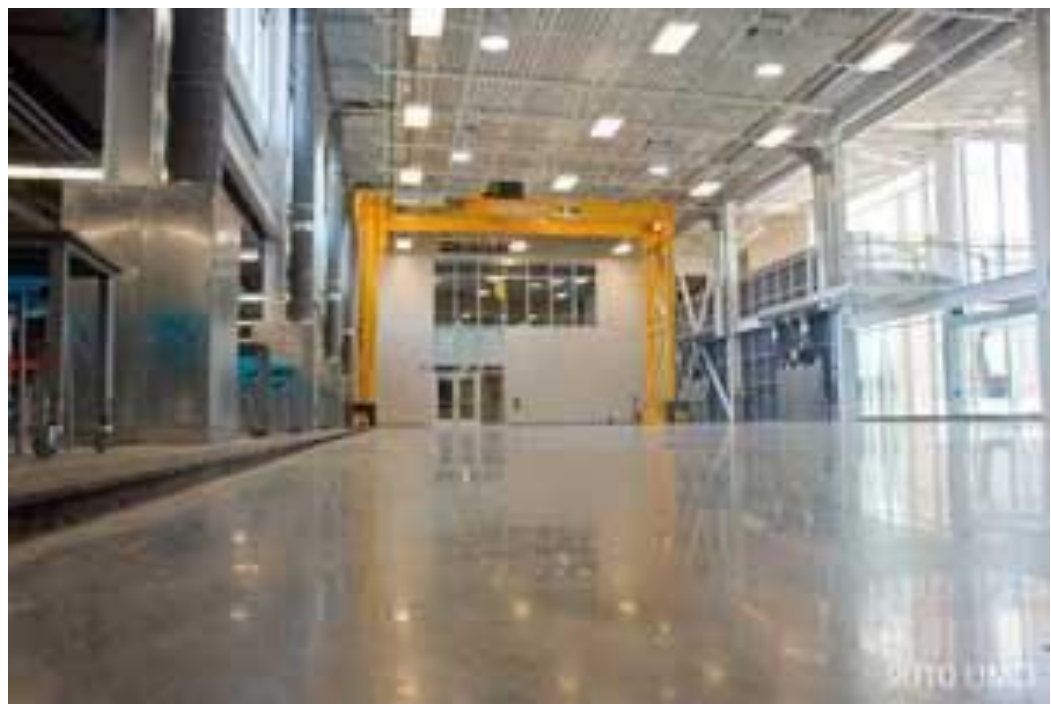

Figure 1: 15 ton gantry crane located in the high bay lab of the Civil Engineering building

\footnotetext{
${ }^{1}$ RISA 2D, educational version, http://www.risa.com/products.html
} 


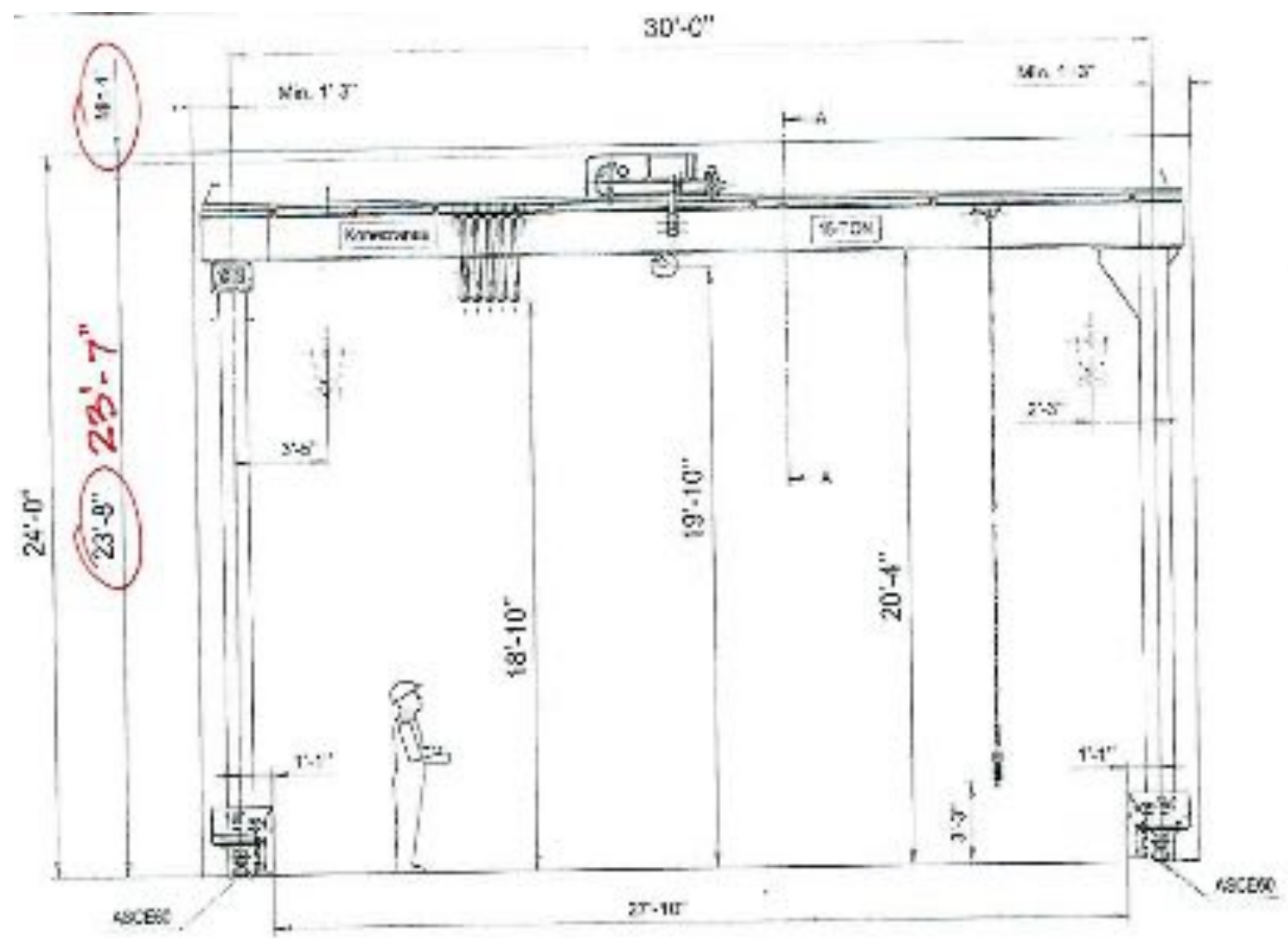

Figure 2: Schematic drawing showing the preliminary design for the gantry crane, including dimensions.

\section{Gantry Crane Project Assignment}

The first task of the project was to create an idealization of the structure. Students were asked to represent the three-dimensional structure as two-dimensional. They were asked to make assumptions about loading, supports and connections that would simplify the structure so that it would resemble a 2D frame such as those appearing in the course text ${ }^{2}$. This first step of the analysis procedure highlights the challenges involved in analyzing actual structures, rather than textbook examples. The students were encouraged to apply their knowledge of structural engineering to make observations about the design of the structure. For example, the fixed connection on one side of the crane bridge allows the structure to carry moment in that connection, and it can be readily observed that the structure is designed to have a greater cross section in the region where it carries this moment. An initial class discussion was held to reinforce these ideas, and to make sure the idealization of the structure was correct, prior to the students beginning the next phase of the project.

The students were asked to use the force method of analysis and the displacement method

${ }^{2}$ Hibbeler, R.C. "Structural Analysis," 7th ed., Prentice Hall, 2008. 
of analysis, two topics studied extensively earlier in the semester, to quantify the behavior of the structure under load. These quantities include support reactions and shear and moment diagrams. The students were required to submit a professional quality report, including a verification of their hand calculations using the modeling software RISA.

In order to familiarize the students with RISA, a lecture was devoted to the use of the software, its applications, and the prevalent use of RISA and similar computer programs to solve engineering problems. The utility of the computational approach was underscored when the students were able to compare the relative ease of application with that of their own hand calculations. A sample of the output generated by the RISA software is shown in Figure 3.

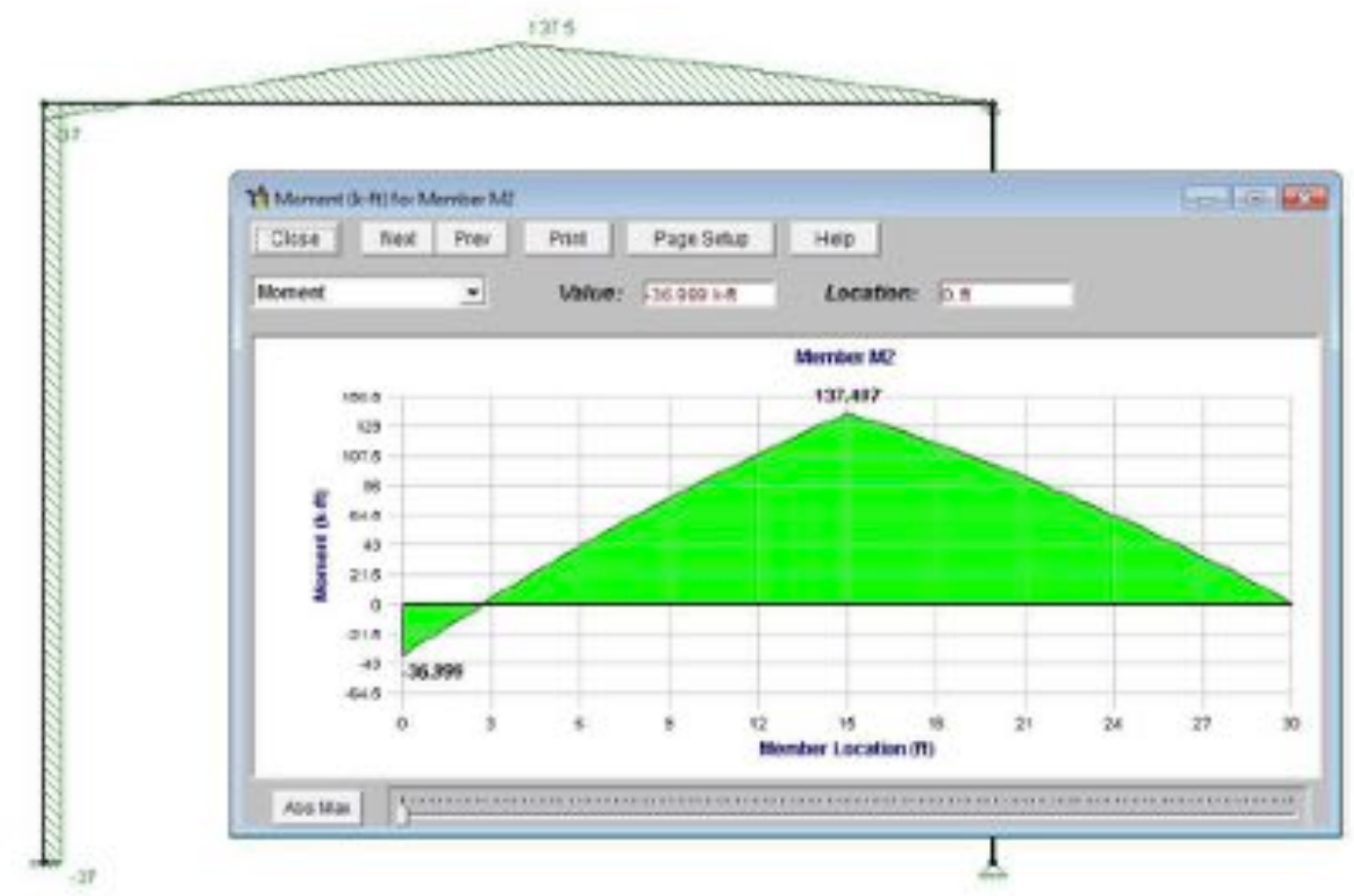

Figure 3: RISA generated moment diagram showing the moments generated when the crane is loaded with 15 tons at midspan.

The final part of the project involved the construction of influence lines. This topic was covered extensively in lecture and was found to be conceptually challenging for many students, particularly the concept of qualitative influence lines. It has been shown that the qualitative influence line may be scaled by a scale factor to recover a true influence line for the structure. Students were asked to use RISA software to develop a quantitative influence line and determine the scale factor, then plot the actual influence line using this data. 


\section{Evaluation}

Grades were assigned by taking into account the objectives of the project. The students were to apply knowledge of mathematics, science and engineering; accuracy of the results was emphasized. This emphasis was particularly placed on conceptual understanding; for example checking that the correct signs appeared on the moment diagrams denoting tension and compression, and that the statically indeterminate structure (unlike a determinate structure) was associated with nonlinear moment diagrams.

The student's ability to communicate effectively was also evaluated; each report was evaluated for clarity, organization and effective presentation. Students were evaluated on their ability to use the techniques, skills and modern engineering tools necessary for engineering practice. They are expected to be able to perform both hand calculations and also be proficient at using software to evaluate the behavior of a structure under load.

The mean score on the project was an $88.4 \%$. In general, the students performed well on the hand calculations and showed an understanding of the use of RISA modeling software. The part of the project that required calculation of actual influence lines based on qualitative influence lines was the most challenging, and many students performed incorrect calculations.

\section{Conclusions}

The fall semester of 2010 was the first semester in which this project was assigned. The project is considered successful; students were able to apply their knowledge of structural analysis to a hands-on problem. The goal for future semesters is to improve the effectiveness of the project as a tool for helping students to integrate knowledge from different topics presented in the Structural Analysis course. To this end, several revisions to the assignment are under consideration. First, the in-class RISA tutorial will be improved through use of the 3D RISA modeling software available to students in the computer lab. In the previous project, 2D RISA software was used. Though the 2D software is more straightforward for a 2D structure, the use of 3D software will better prepare students to perform 3D analysis in other courses in the structural engineering focus area.

Though most of the project topics were clearly understood by the students, further clarification is required in the portion of the project calling for the construction of influence lines based on qualitative influence lines. Further explanation in lecture is required to allow students to understand the procedure fully. It may be useful to approach influence lines by direct calculation prior to construction using qualitative influence lines.

The gantry crane project was beneficial in helping the students integrate different topics in the course that can be applied to the analysis of a single structure. The calculation of support reactions, and moment and shear internal loads has a clear relation to the design 
parameters for the structure and its supports. If the project is to be extended, the calculation of stresses may be performed and the crane may be evaluated from a design perspective.

KATHERINE ACTON is an assistant professor at the University of Minnesota Duluth. Her areas of teaching interest include Structural Analysis, Mechanics, and Finite Element Methods. Her research focus is in computational solid mechanics. She earned her doctorate from Johns Hopkins University in 2009. 